\title{
The Research on Delay Propagation of Urban Rail Transit Operation under Sudden Failure
}

\author{
Yuan Zhao' ${ }^{1}$ and Xiaobing Ding $\mathbb{D}^{2}$ \\ ${ }^{1}$ The Key Laboratory of Road and Traffic Engineering, Tongji University, Shanghai 201804, China \\ ${ }^{2}$ School of Marine and Architectural Engineering, Shanghai Jiaotong University, Shanghai 200240, China \\ Correspondence should be addressed to Xiaobing Ding; dxbsuda@163.com
}

Received 8 April 2021; Accepted 3 May 2021; Published 15 May 2021

Academic Editor: Xinqiang Chen

Copyright (c) 2021 Yuan Zhao and Xiaobing Ding. This is an open access article distributed under the Creative Commons Attribution License, which permits unrestricted use, distribution, and reproduction in any medium, provided the original work is properly cited.

\begin{abstract}
With the increase and extension of urban rail transit lines, networked operation has become an inevitable trend of rail transit operations. Once an emergency occurs, it will cause operational delays; in serious cases, it may further lead to group safety incidents. Firstly, the sudden failure of rail transit is defined, statistical calculation is made according to the accumulated failure data, and then the sudden failures and average processing time are quantitatively calculated. Secondly, the time delay and propagation under the state of sudden failure are analyzed, on the basis of which the propagation and dissipation of time delay based on the single station failure cellular automata model and SIR model network based on multistation fault delay propagation are constructed. Finally, the reliability and accuracy of the model are verified by a case of rail transit in a city. The scheme in this paper can be used to estimate the scope of time and space delay under the sudden failure of rail transit and can provide the basis for the adjustment of traffic organization scheme and evacuation of passenger flow under the sudden failure.
\end{abstract}

\section{Introduction}

Urban rail transit network operation involves many types of facilities and equipment, strong interaction, and strong linkage effects between stations and lines. Once an operation accident occurs, the train operation delay will affect the entire network. The delayed line not only reduces its passing capacity but also causes some passengers to change the travel route, which increases the probability of delays due to oversaturation of passenger flow on other lines, causing the delay to spread rapidly. The network operation of urban rail transit effectively improves the service level and resource utilization, and the network scale effect attracts more passenger flow, but, at the same time, the network operation increases the difficulty of management and increases the operation load. There are many kinds of facilities and equipment in urban rail transit network, which have wide mutual influence. The linkage effect between stations and lines is strong. Once the operation accident (failure) occurs, the train operation delay will gradually affect the whole road network. The delay of urban rail transit line will not only reduce the carrying capacity of the line but also affect the subsequent trains entering the line. At the same time, the delay may be transmitted to the terminal station and affect the train turn back (except the loop line), so as to transfer the delay to the other direction. In the state of delay, the line not only reduces its own carrying capacity but also causes some passengers to change their travel path, which increases the probability of delay of other lines due to passenger flow saturation, resulting in delay propagation. For example, the passenger flow delay caused by signal failure of Metro Line 10 in a city has caused delay ripple effect on Line 6, Line 13, Line 1, and Line 15. After the metro network is put into operation, the delay propagation and ripple effect will be more obvious. Therefore, it is necessary to study the quantitative analysis method of train operation delay and its propagation range based on the characteristics of different faults, so as to guide the operation units to do a good job in prevention and emergency treatment in time and minimize the impact of delay. 
Some scholars at home and abroad have done relevant research on the law of the propagation of fault delays and the influence of passenger flow: Zhang et al. [1] used cellular automata to establish the evolution rules of the local interaction of passenger flow and quantitatively analyzed the rail transit network layer under the large commuter flow. When the sudden fault occurs, the passenger flow presents different propagation rules in the two angles of line and station. Zhang et al. [2] analyzed the vulnerability of large and complex rail transit networks and calculated their recovery speed and designed a large and complex rail transit network recovery evaluation framework. Ding et al. [3] designed a rail transit dispatching fault log management and data database system for rail transit operation fault analysis and early warning and has a more accurate prediction and judgment on the delay time of various operation faults. Gao et al. [4] constructed an optimization model for train dispatching when subway lines are interrupted and a large number of passengers are stuck in stations. The effect of dispatching will directly affect the dissipation of rail transit failures and delays. $\mathrm{Xu}$ et al. [5] established an integer programming model with the goal of minimizing the total travel time of all passengers and constructed a combined dynamic programming algorithm with multitrain and multistation time adjustment. Ye et al. [6] analyzed the influence of train delays on the choice of passenger travel mode, gave the judgment condition formulas for passenger flow affected by train delays, and gave the calculation model of travel passenger flow in three modes. Chen [7] proposed an augmented ship tracking framework via the kernelized correlation filter (KCF) and curve fitting algorithm, which can successfully track ships in maritime video clips with high accuracy, and it can significantly outperform the other popular ship trackers.

In summary, domestic and foreign research on the propagation of rail transit delays mainly focuses on sudden large passenger flows or commuting large passenger flows under single-line conditions and rarely considers the impact of the following train operation delays on the network under sudden failure conditions. Some scholars focus on building a small-world network model to simulate congestion propagation [8], without considering the actual road network conditions of rail transit, and the accuracy is low. There are also documents that use the SIS virus infection model to simulate the spread of congestion [9], but it does not involve the interaction of the delay status between stations and can only vaguely predict the number of delayed stations. Chen [10] proposed a multiview learning algorithm to extract a highly coupled and robust ship descriptor from multiple distinct ship feature sets and proposed a framework for integrating a multiview learning algorithm and a sparse representation method to track ships efficiently and effectively.

This paper constructs a rail transit delay propagation model, uses the cellular automata model and the SIR model to simulate the delay propagation rule, and predicts the delay time rule. Finally, the feasibility and accuracy of the model are verified by the simulation analysis of a certain urban rail transit network.

\section{Problem Description}

After the failure of rail transit, due to the time required for fault identification, information transmission, and fault treatment, the operation management department needs to predict the initial time of the failure and deeply study the propagation of delay caused by the failure according to the time period, line, and interval of the failure.

\section{Analysis on Train Operation Delay under Sudden Failure of Urban Rail Transit}

3.1. Sudden Failure of Urban Rail Transit. There are many types of failures that cause delays in train operation, and the frequency and duration of their occurrence vary greatly. In order to study the law of its occurrence, a set of calculation methods are based on the original data of the dispatch log, deredundancy, and desensitization, and then the time, space, reason, and other parameters of the fault delay are calculated [11-14]. Taking the dispatch log of a rail transit network in 2018 as an example, there are a total of 13,423 valid data items, 26 subcategories are processed, and the occurrence frequency and proportion are calculated, and the fault subcategories are merged into major categories to obtain the fault distribution and average processing time as shown in Table 1.

\subsection{Train Operation Delay under Sudden Failure of Urban Rail} Transit. The spread of train delays includes spread in space and delays in time. Under ideal conditions, the passengers of the preceding vehicle transfer to the connecting train through the transfer station, and the corresponding redundant time will be reserved in the train operation diagram [15-17]. The preceding vehicle has a delay, and the delay can be dissipated through the redundant time of the connecting train, and the running time of the section can be changed by adjusting the running speed of the section to reduce the time impact caused by the delay. If the preceding train is delayed at the platform and the original stop redundancy time cannot meet the passenger demand, the subsequent train stopping time $\left(T_{\mathrm{S}}\right)$ can be adjusted and shortened. If the sum of the running time $\left(T_{\mathrm{R}}\right)$ and the stopping time cannot compensate for the delay caused by the failure, the delay will be transmitted to the train line and the entire network through the train [18-21].

In this paper, the train delay can be decomposed into two parts in terms of time: the handling time of the sudden failure itself and the consequent delay time. Taking Figure 1 as an example, a sudden failure occurs at station $\mathrm{A}$, and the fault processing time is $T_{i}$. Using interval running time and stop redundancy time, station $G$ (travel time from A to station G) will have a delay of $T$ after $\sum t_{n}$ [22]. Due to the use of section operation and stop redundancy time, the delay time will be less than $T_{i}, T \leq T_{i}$. Continuing this adjustment, if the initial delay $T_{i}$ is not large, the initial delay caused by the fault can be compensated.

According to the line and time period of the sudden failure, the initial time can be obtained according to the 
initial failure category, and the delay time of the affected station can be studied by combining the interval running time and the difference of the train stopping time, as shown in the following equation:

$$
T_{0}=T_{R}+T_{S},
$$

where $T_{R}$ is train running time; $T_{S}$ is train stopping time.

$$
T_{0}^{\prime}=T_{i} \times w_{i j}+T_{R}^{\prime}+T_{S}^{\prime}=T_{i} \times \frac{k_{i}}{k_{i}+k_{j}} \times l_{i, j} \times w_{\text {time }}+T_{R}^{\prime}+T_{S}^{\prime} .
$$

The calculation in equation (2) involves three quantities, which are based on the structure of the road network, the importance of the passenger flow of the faulty line, and the period of time when the fault occurs to build the model. Among them, the ratio of the structural importance of station $i$ to the average importance of the road network structure in the interval from station $i$ to station $j$ reflects the structural importance of the faulty station $i$ in the faultaffected interval; $l_{i, j}$ reflects the importance of the fault line in the whole network line; $w_{\text {time }}$ is determined by the time period of the fault occurrence and is mainly obtained based on the proportion of the passenger flow volume in the time period to the entire network. $T_{0}$ is running time without delay; $T_{i}$ is fault handling time. $T_{0}$ ' is time in case of delay. $w_{i j}$ is comprehensive weight of the faulty station in the faulty road network, where $W_{i j}=\left(k_{i} / k_{i}+k_{j}\right) \times l_{i, j} \times w_{\text {time }} ; T_{R}^{\prime}$ is the actual running time after removing the redundant running time of the train section: $T_{R}^{\prime} \leq T_{R}$. $T_{S}^{\prime}$ is the actual stopping time of the train after the redundant stop time is removed. $T_{S}^{\prime} \leq T_{S} . k_{i}$ is the importance of the road network structure of station $i$ in the road network. Consider the value of $k_{i}$ only from the perspective of the road network structure: $k_{i}=2^{*} N_{L}$, and $N_{L}$ is the number of transferable lines at station $i$. If it is a nontransfer station, take 1 ; if it is a transfer station, it will be calculated according to the number of transfer lines, and so on. $k_{j}$ is the importance of the road network structure of station $j$ in the road network. $l_{i, j}$ is the weight of the faulty line in the road network, which is derived from the ratio of the total daily average passenger flow from station $i$ to station $j$ of the line to the average total passenger flow of the entire network; namely, $l_{i j}=p_{i j} / p_{0}$, where $p_{i j}$ is the total daily average passenger flow from station $i$ to station $j$ of the line and $p_{0}$ is the average total passenger flow of the entire network. $w_{\text {time }}$ is the converted weight of the time period when the fault occurs based on the proportion of the amount of passenger flow in the time period to the entire network, and the value is shown in Table 2.

In equation (2), $T_{R}$ is determined by the interval distance and interval running speed. When the train is in a delay state, the delay time can be restored by increasing the interval running speed. $T_{S}$ can be dynamically adjusted according to the passenger flow of the station to dissipate delays to a certain extent.

Table 2 shows the converted weights based on the time period, mainly based on the average passenger flow data of a subway line from March 28, 2019, to April 3, 2019. Take the inbound passenger flow $P_{i}$ during each time period and the average total inbound passenger flow $P_{t}$ during the operating period; $w_{\text {time }}=P_{i} / P_{t}$, where, $P_{i}$ and $P_{t}$ are obtained from AFC data. After data cleaning and calculation, the converted weights based on the time period in Table 2 can be obtained.

3.3. Dissipation Modeling of Train Delays. The Bass model was proposed by Frank M. Bass [9] in 1996 to study an important model of the diffusion mechanism in the life cycle, and a series of improved models emerged on this basis [10]. The propagation of rail transit failure delays is similar to the demand diffusion of short-life cycle products in the market. The dynamic behavior of rail transit network passenger flow transfer can be simulated through the complex network topology of rail transit as shown in the following equation:

$$
N_{t}=N_{t-1}+\alpha_{i j}\left(m-N_{t-1}\right)+w_{i}^{j} \frac{N_{t-1}}{m}\left(m-N_{t-1}\right),
$$

where $N_{t}-t$ is the number of stations affected by delays at any time; $\alpha_{i j}$ is delay propagation coefficient due to passenger flow transfer from station $i$ to station $j ; m$ is the maximum number of stations that may be involved in transmission due to delays. The value of $m$ is calculated according to the structural importance of the faulty station in the road network, and $W_{\text {time }}$ weighting calculation in Table 2 of the comprehensive fault occurrence time period. The calculation process is as follows:

$$
m=\frac{1}{2} \frac{k_{i}}{1 / n \sum_{i=1}^{n} k_{i}} \times w_{\text {time }},
$$

where $n$ is the total number of stations on the line where the fault occurred.

$$
\alpha_{i j}=\frac{p_{0}+p_{j}-q_{j}}{h_{l}},
$$

where $p_{0}$ is the number of stranded passengers at station $j$, the data of stranded passengers at station $j$ can be obtained through Wi-Fi probe device, and the acquired Wi-Fi collected data can be processed to obtain $P_{0} \cdot p_{j}$ is the number of people getting on the train at station $j ; q_{j}$ is the number of people getting off the train at the station; $h_{l}$ is the transport capacity of train $l$.

After a sudden failure in rail transit, while the delay spreads at a certain speed, passengers will choose to transfer to other modes or routes, so that the delay will dissipate at a certain speed at the same time. In order to characterize this passenger flow dissipation, $\beta_{\mathrm{ij}}$ is introduced to construct the delay. The dissipation model is shown in the following formula:

$$
L_{t}=N_{t-1}-\beta_{i j} N_{t-1}-w_{i j} \frac{m-N_{t-1}}{m} N_{t-1}
$$

where $L_{t}$ is the number of stations where the delay dissipated; $\beta_{i j}$ is the proportion of passenger flow dissipated due to passenger flow to other transportation modes; $\beta_{i j}=p_{s} / h_{l}$, where $p_{s}$ represents the amount of passenger flow dissipated from passenger flow to other transportation modes. 
$N_{t}$ expresses the number of stations affected by delays at time $t$. With the passage of time and repair operations, the loss of passenger flow, and other reasons, $N_{t}$ gradually decreases; and $L_{t}$ expresses the number of stations whose delays dissipate at time $t$, the number of delayed trains is gradually decreasing. $N_{t}$ and $L_{t}$ describe the characteristics of delay propagation and dissipation in the road network from two perspectives.

\section{Propagation Modeling of Sudden Failures in following Vehicle Operation}

4.1. Network Delay Propagation Based on Cellular Automata under Station Failure. In order to predict the average trend of delay propagation under sudden failures, modeling is needed to characterize the interaction mechanism between stations in the delay state $[23,24]$. The cellular automata model has a good ability to simulate the evolution of time and space and is more consistent with the study of the delay propagation of a single line and a single station. The following assumptions are made:
(1) The station is used as the cell, and the road network is used as the cell space to distinguish the upper and lower lines

(2) Take the actual departure interval as the step length, and the state at time only affects the state at time

(3) The number of affected stations is less than the total number of stations

Determine the state of the station at the next moment through the current state of the station and use $S_{j}(t)$ to represent the state of station $j$ at time $t$; then, $S_{j}(t+1)=f\left(S_{j}(t), \alpha_{i j}(t), \beta_{i j}(t), w_{i j}(t)\right)$. According to the established transfer function model, when there is a sudden failure, if the number of waiting passengers accumulated in station $j$ is much greater than the number of people getting off the train at the station, and the station is overloaded, the delay will propagate to neighboring stations; otherwise, the delay will be transmitted to neighboring stations. Dissipate to neighboring stations, and simulate the influence mechanism into a model shown in the following equation:

$$
S_{j}(t+1)=\left\{\begin{array}{ll}
X_{j} & \sum t_{n} \leq T \leq \sum t_{n}+T_{1} \\
S_{j}(t)+\frac{p_{j}-q_{j}}{h_{l}}+\frac{p_{j}-q_{j}}{\left|p_{j}-q_{j}\right|} \frac{k_{i}}{k_{i}+k_{j}} \frac{S_{j}(t)}{S_{j}(t)+1} & T>\sum t_{n}+T_{1}
\end{array},\right.
$$

where $X_{j}=\left\{\begin{array}{ll}0 & \text { station } j \text { open to traffic } \\ 1 & \text { station } j \text { closed to traffic }\end{array}\right.$, and when $s(t) \in[0,1]$, the station is in normal condition; when $S(t)>l$, the station is in delayed status. $t_{n}$ is delay time of each station, and $t_{n}$ can be calculated by formula (2) in turn; $T_{1}$ is initial delay time; $T$ is current delay time: $T=T_{0}^{\prime}-T_{0}$.

$S_{j}(t)$ indicates the status of the station at time; when $S(t) \in[0,1]$, the station is in normal condition; when $S(t)>1$, the station is in delayed status. According to the constructed network delay propagation model based on cellular automata under station failures, it is possible to determine the subdelay dissipating period of the affected station and the delay status of the station and quickly and accurately determine whether the station is delayed or dissipated.

4.2. Train Operation Delay Based on SIR Model in the Case of Multiple Station Failures. In the actual operation of urban rail transit, the "delay" node propagates along with the direction of the train and other trains affected by the delay. The Susceptible Infected Recovered Model (SIR model for short) is a transmission model, in which $\mathrm{S}$ represents susceptible persons, I represents infected persons, and $R$ represents evacuated persons, which can well describe the spread of multistation delays [25].

The SIR model is used to simulate the propagation of operation delays in the state of sudden failures at multiple stations in the road network at the same time, and the established dynamic differential equation is the following equation:

$$
\left\{\begin{array}{l}
\frac{d s(t)}{d t}=-\alpha i(t) s(t) \\
\frac{d i(t)}{d t}=\alpha i(t) s(t)-\beta i(t), \\
\frac{d r(t)}{d t}=\beta i(t)
\end{array}\right.
$$

where $s(t), i(t), r(t)$ denote proportion of normal stations, delayed stations, and restored stations; $\alpha$ denotes delay spread rate; $\beta$ denotes delay dissipation rate.

Discretize the delay propagation model according to formula (8), which can be transformed into the following formula:

$$
\left\{\begin{array}{l}
\Delta S_{t+1}=-\alpha I_{t} S_{t} \\
\Delta I_{t+1}=\alpha I_{t} S_{t}-\beta I_{t} \\
\Delta R_{t+1}=\beta I_{t}
\end{array}\right.
$$

where $S_{t}$ denotes the number of stations that may be delayed at time $t ; I_{t}$ denotes the number of stations delayed at time $t$; $R_{t}-t$ denotes the number of stations where the delay dissipated at time $t$. 
TABLE 1: Operation delay of urban rail transit.

\begin{tabular}{|c|c|c|c|c|}
\hline Main category & Subcategory & $\begin{array}{l}\text { Frequency } \\
\text { (times/year) }\end{array}$ & $\begin{array}{l}\text { Average } \\
\text { processing time } \\
(\mathrm{min})\end{array}$ & $\begin{array}{l}\text { Proportion } \\
\quad(\%)\end{array}$ \\
\hline Train factors & $\begin{array}{c}\text { Train failure, door, auxiliary control circuit, control circuit, } \\
\text { brake, main circuit }\end{array}$ & 127 & 17.6 & 30.75 \\
\hline $\begin{array}{l}\text { Communication signal } \\
\text { factor }\end{array}$ & $\begin{array}{c}\text { ATS, vehicle ATC, trackside equipment, communication, } \\
\text { signal, etc. }\end{array}$ & 184 & 18.2 & 44.55 \\
\hline Power supply factors & Substation, touch network, power supply, etc. & 8 & 23.4 & 1.94 \\
\hline Public works factors & Turnouts, lines & 22 & 7.6 & 5.33 \\
\hline $\begin{array}{l}\text { Passenger } \\
\text { transportation factors }\end{array}$ & $\begin{array}{c}\text { Screen door/safety door, driver delay, passenger } \\
\text { transportation, and others }\end{array}$ & 14 & 14.3 & 3.39 \\
\hline Objective factors & $\begin{array}{c}\text { Passengers, people and objects, intrusion limits, large } \\
\text { passenger flow, personnel invasion, human-train conflict, } \\
\text { objectives, and others }\end{array}$ & 58 & 3.3 & 14.04 \\
\hline
\end{tabular}

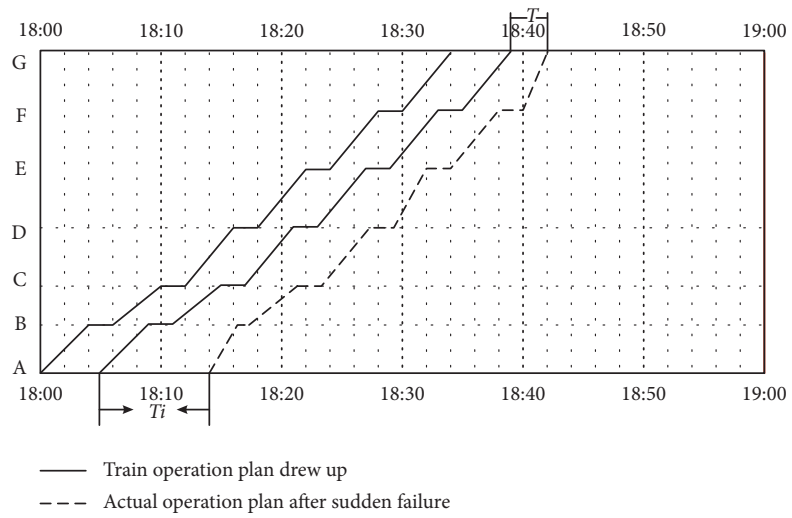

Figure 1: The operating chart of traffic delay dissemination. Train operation plan drew up. Actual operation plan after sudden failure.

TABLe 2: Time period discount weight based on time period division $\left(w_{\text {time }}\right)$.

\begin{tabular}{lccccc}
\hline Time period & $7: 00-9: 00$ & $9: 00-17: 00$ & $17: 00-19: 00$ & $19: 00-21: 00$ & Others \\
\hline$w_{\text {time }}$ & 0.31 & 0.12 & 0.28 & 0.12 \\
\hline
\end{tabular}

4.3. Time and Space Propagation of Train Operation Delay. Based on the average processing time $T_{\mathrm{i}}$ of the major types of sudden failures in Table 1, comprehensive lines, fault occurrence time periods, and so forth, substitute equation (3) to get the amount of station delay $N_{t}$. According to formula (1) and formula (2), calculate $T$ under this type of fault as follows:

$$
T=T_{0}^{\prime}-T_{0} .
$$

In the end, the number of delayed stations $N_{t}$ and the time delay $T_{0}^{\prime}$ can be estimated according to the major types of failures, as shown in Table 3.

\section{Case Analysis}

5.1. Estimation of Delay Time under Sudden Failure Conditions. Take Shanghai Metro Line 9 (Guilin Road Station-Middle Yanggao Road Station), Line 2 (Weining Road Station-Shanghai Science and Technology Museum Station), Line 7 (Langao Road Station-Longhua Middle Road Station), a total of 116 stations, and 51 nodes are the research objects. Take the vehicle breakdown on Zhaojiabang Road of Line 9 as an example to study the propagation and dissipation process of delays in the road network during the 8:00-9:00 a.m. period [26]; the schematic diagram of Shanghai subway network structure can be shown in Figure 2.

The initial delay of the train failure and formulas (4) and (5) calculate the initial delay propagation rate and dissipation rate. The initial failure processing time is $19.1 \mathrm{~min}$. The delay propagation and dissipation of the road network are simulated by Matlab.

The calculation results of the example (as shown in Table 4) show that, after the delay occurs, it spreads in both directions in the section or the single line. The transfer station plays an initial role of evacuation. As the delay time increases, it gradually spreads to the adjacent line.

5.2. Propagation and Dissipation Where Delays Occur at a Single Hub. Assuming that there is a power failure on Zhaojiabang Road and Century Avenue of Line 9, the initial 
TABLE 3: Time and space propagation of train operation delay due to sudden failure.

\begin{tabular}{lccc}
\hline Sudden failure (category) & Processing time $(\min ), T_{i}$ & Delay station, $N_{t}$ & Delay time $(\mathrm{min}), T$ \\
\hline Train factor & 17.6 & 8 seats & 41.8 \\
Power supply factor & 20.5 & 22 seats & 93.2 \\
Public works factor & 7.1 & 5 seats & 23.1 \\
Communication signal factors & 18.2 & 16 seats & 59.5 \\
Passenger factor & 14.3 & 9 seats & 35.7 \\
Objective factor & 3.3 & 4 seats & 19.6 \\
\hline
\end{tabular}

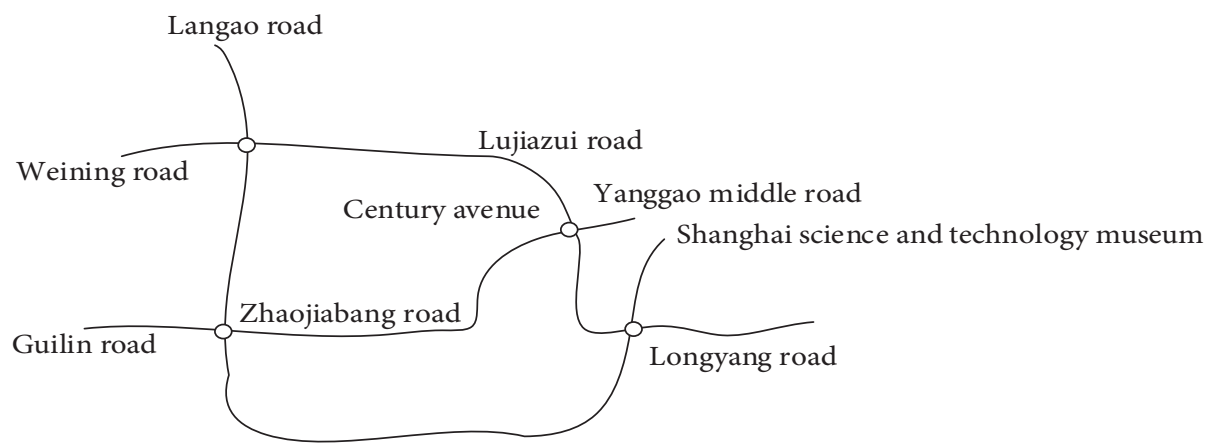

FIgURE 2: Schematic diagram of Shanghai subway network structure.

delay is $21.25 \mathrm{~min}$, the importance of Zhaojiabang Road Station is $k=4$, and the importance of Century Avenue Station is $k=8$. The delay propagation rates of the two stations are 0.45 and 0.54 , respectively, and the dissipation rates are about 0.094 and 0.099 . The amount of station delay is shown in Table 5 and Table 6.

It can be seen from Table 4 that the initial delay of 8.11 min was imposed on the train on Zhaojiabang Road, and Line 9 departed at an average departure interval of $3.82 \mathrm{~min}$. The delay affected the following 7 trains. At the initial time of the fault $(0 \mathrm{~min})$ and the second train departure (the average departure interval is $3.82 \mathrm{~min}$ ), the simulation results of the number of delayed stations are shown in Figure 3 (the simulation at other times is limited to the space for the time being).

Figures 3(a) and 3(b) are the simulation diagrams of delay estimation and dissipation in the case of power supply failure in Zhaojiabang Road and Century Avenue of Shanghai Rail Transit Line 9.

In Figure 4, the blue “-” graph represents the number of stations that may be delayed, the orange "*” represents the number of stations that are delayed, and the yellow "+" represents the number of stations where delay dissipates. When the initial delay occurs on Zhaojiabang Road, the maximum number of delayed stations is 28 . When the initial delay occurs on Century Avenue, the maximum number of delayed stations is 30 . It can be seen that if it is a large transfer station, due to the connection of many lines, once there is a delay, it will spread in the transfer line until it affects the whole network.

When the delay occurs on the same line and the same type of station, the propagation rate and dissipation rate of the delay remain stable, and the number of delayed stations will not increase significantly with the initial number of delayed stations. Comparing the simulation results with the historical delay data of Zhaojiabang Road down Line 9 shows that the time-space propagation range basically conforms to the actual delay situation, which verifies the validity of the built model.

5.3. Propagation and Dissipation Where Delays Occur at Multiple Hub Stations. When delays occur at multiple stations, which may occur at the same time due to various events, in particular, when terrorist attacks and natural disasters occur, multiple stations in a certain section will be affected and be unable to normally operate, causing a greater impact. Tables 7 and 9 show the multistation delay based on SIR model simulation and, respectively, study the situation where $k=2$, and there are 5,10 , and 15 intermediate stations of Line 9 fall failure, the delay propagation, and dissipation when the initial delay occurs.

Figure 5 shows the simulation of simultaneous delays at multiple stations based on the SIR model. Under the condition of $k=2,5,10$, and 15 intermediate stations of Line 9 have vehicle failures, and the delay propagation and dissipation when the initial delay occurs. From Figures 5(a) and 5 (c), it can be seen that when the delay occurs on the same line and the same type of station, the propagation rate and dissipation rate of the delay remain stable, and the number of delayed stations will not increase significantly with the initial number of delayed stations. Comparing the simulation results with the historical delay data on Zhaojiabang Road down Line 9 shows that the time-space propagation range basically conforms to the actual delay situation, which verifies the validity of the built model. 
TABLE 4: Delay time and spread scope of vehicle failure in Zhaojiabang station (part).

\begin{tabular}{lcc}
\hline Station & Delay period & Maximum delay (min) \\
\hline Zhaojiabang Road (down) & $8: 00: 00-8: 23: 24$ & 8.11 \\
Jiashan Road (down) & $8: 02: 00-8: 23: 48$ & 7.73 \\
Century Avenue (down) & $8: 17: 00-8: 53: 46$ & 2.68 \\
Guilin Road (up) & $8: 21: 02-8: 28: 42$ & 4.05 \\
$\ldots$ & $8: 23: 23-8: 38: 40$ & $\ldots$ \\
Yanggao Middle Road (down) & & 2.05 \\
\hline
\end{tabular}

Table 5: Number of stations for network delay propagation and dissipation (Zhaojiabang station fault).

\begin{tabular}{lccc}
\hline Time period & Number of possible delays (seats) & Number of delayed stations (seats) & Number of delays dissipated (seats) \\
\hline $8: 00$ & 57 & 1 & 1 \\
$8: 00-8: 10$ & 19 & 28 & 8 \\
$8: 10-8: 20$ & 22 & 8 & 30 \\
$8: 20-8: 30$ & 12 & 3 & 43 \\
$8: 30-8: 40$ & 6 & 2 & 47 \\
$8: 40-8: 50$ & 2 & 1 & 52 \\
$8: 50-9: 00$ & 1 & 0 & 57 \\
\hline
\end{tabular}

TABLE 6: Number of stations for network delay propagation and dissipation (Century Avenue station fault).

\begin{tabular}{lccc}
\hline Time period & Number of possible delays (seats) & Number of delayed stations (seats) & Number of delays dissipated (seats) \\
\hline $8: 00$ & 58 & 1 & 1 \\
$8: 00-8: 10$ & 25 & 30 & 11 \\
$8: 10-8: 20$ & 2 & 21 & 22 \\
$8: 20-8: 30$ & 1 & 10 & 47 \\
$8: 30-8: 40$ & 0 & 4 & 53 \\
$8: 40-8: 50$ & 0 & 1 & 56 \\
$8: 50-9: 00$ & 0 & 0 & 58 \\
\hline
\end{tabular}

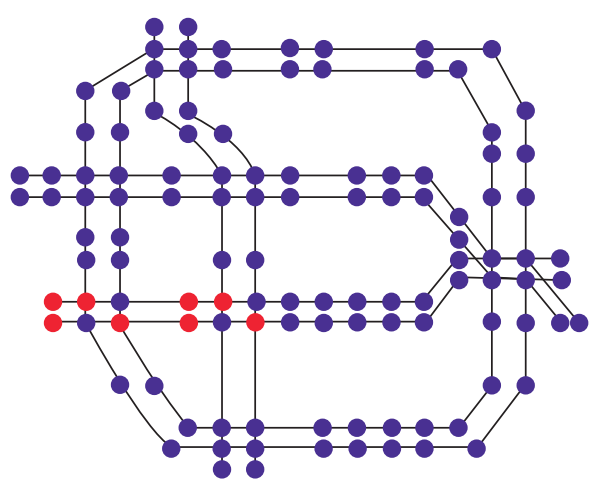

(a)

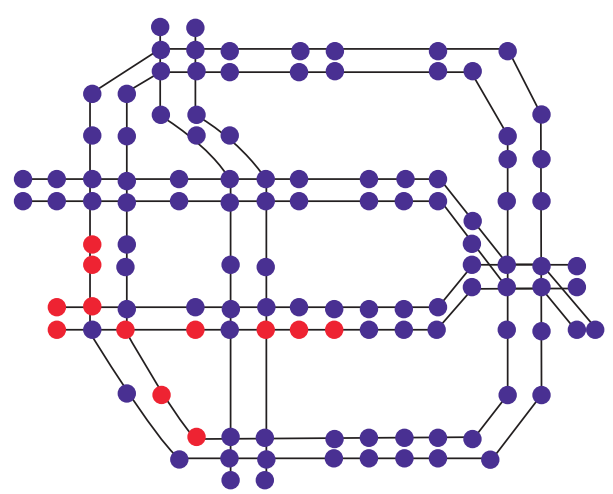

(b)

FIGURE 3: The simulation results of delay propagation in Shanghai urban rail transit network. (a) $T=0 \mathrm{~min}$. (b) $T=3.82 \mathrm{~min}$. 


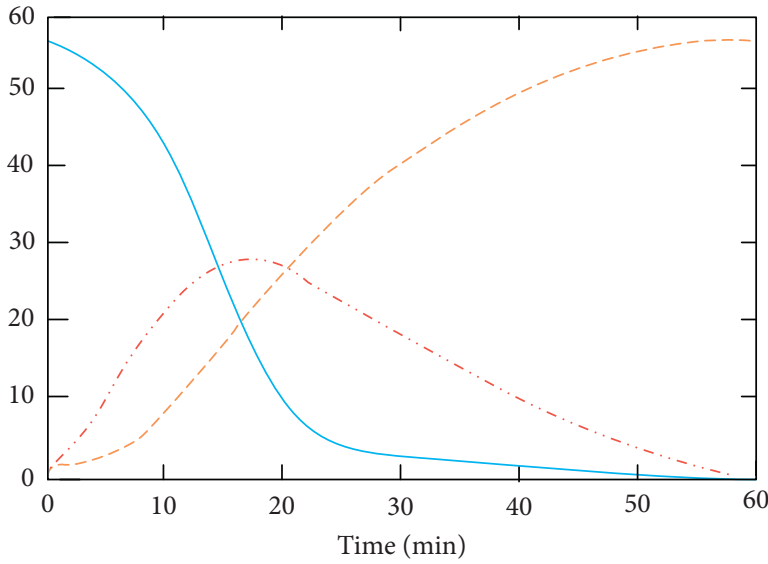

(a)

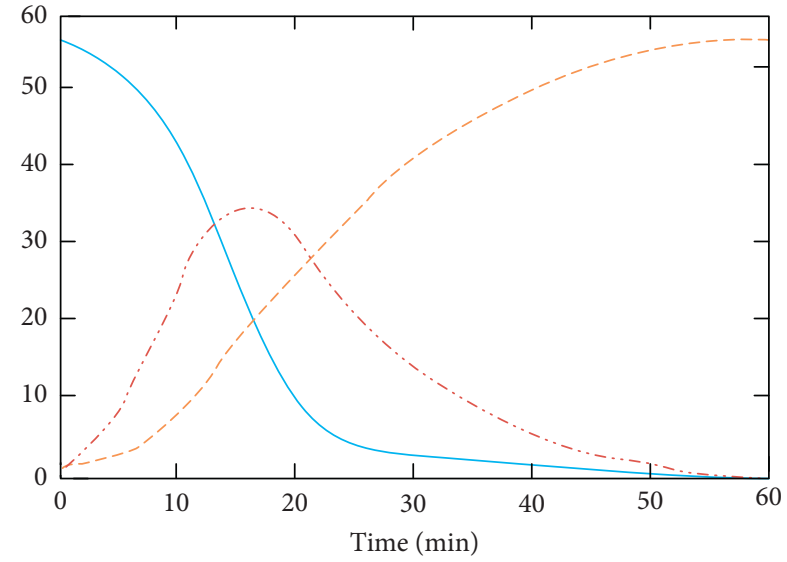

(b)

FIGURE 4: Number of stations for network delay propagation.

TABLE 7: Delay propagation and dissipation in case of 5 stations with degree- 2 vehicle failure.

\begin{tabular}{lccc}
\hline Min & Stations that may be delayed & Stations delayed & Stations with delay dissipated \\
\hline 0 & 52 & 5 & 0 \\
10 & 25 & 25 & 15 \\
20 & 2 & 21 & 35 \\
30 & 1 & 10 & 44 \\
40 & 0 & 4 & 52 \\
50 & 0 & 1 & 55 \\
60 & 0 & 0 & 57 \\
\hline
\end{tabular}

TABLE 8: Delay propagation and dissipation in case of 10 stations with degree- 2 vehicle failure.

\begin{tabular}{lccc}
\hline Min & Stations that may be delayed & Stations delayed & Stations with delay dissipated \\
\hline 0 & 41 & 10 & 0 \\
5 & 22 & 24 & 9 \\
10 & 7 & 20 & 24 \\
15 & 3 & 13 & 34 \\
20 & 1 & 9 & 40 \\
25 & 1 & 4 & 44 \\
30 & 1 & 3 & 47 \\
\hline
\end{tabular}

TABLE 9: delay propagation and dissipation in case of 15 stations with degree-2 vehicle failure.

\begin{tabular}{lccc}
\hline Min & Stations that may be delayed & Stations delayed & Stations with delay dissipated \\
\hline 0 & 36 & 15 & 0 \\
5 & 13 & 26 & 12 \\
10 & 5 & 19 & 26 \\
15 & 2 & 12 & 36 \\
20 & 2 & 8 & 41 \\
25 & 1 & 5 & 45 \\
30 & 1 & 3 & 47 \\
\hline
\end{tabular}




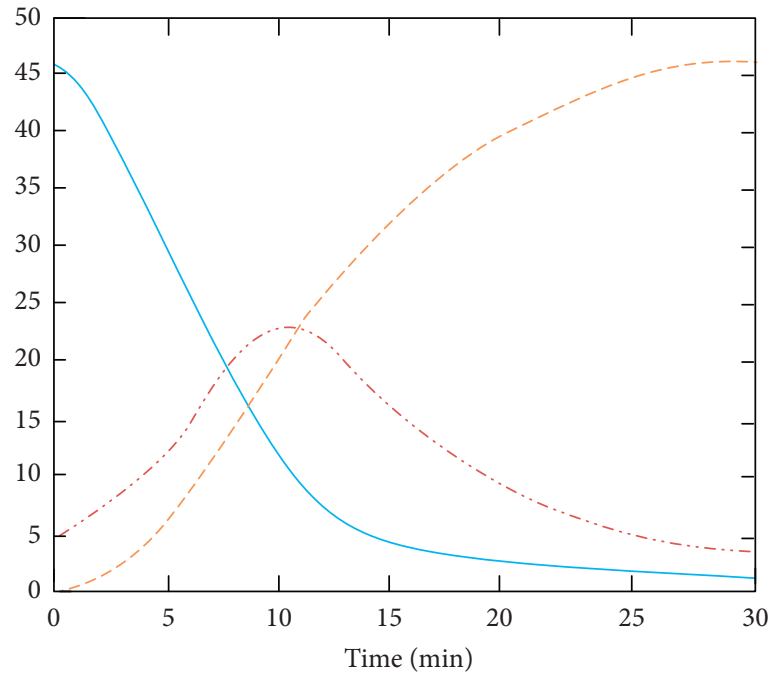

(a)

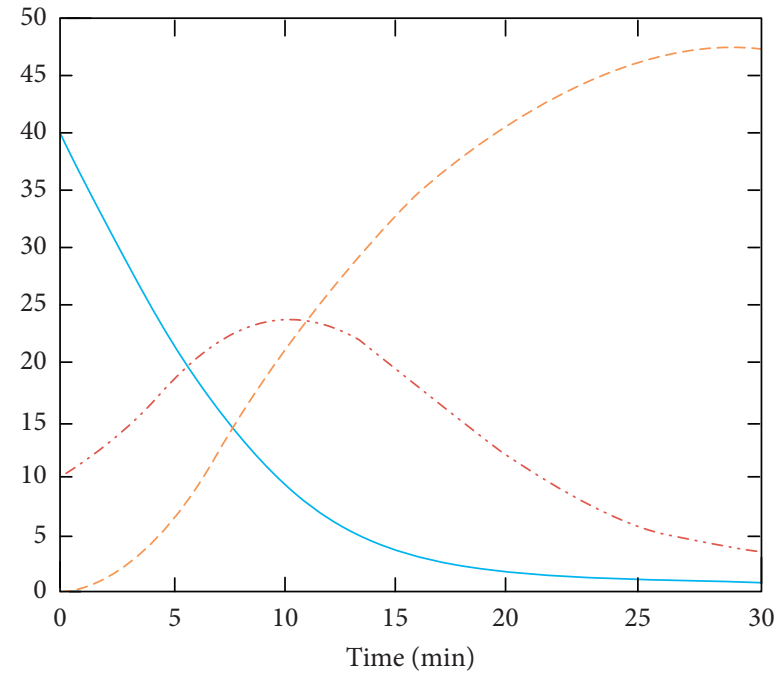

(b)

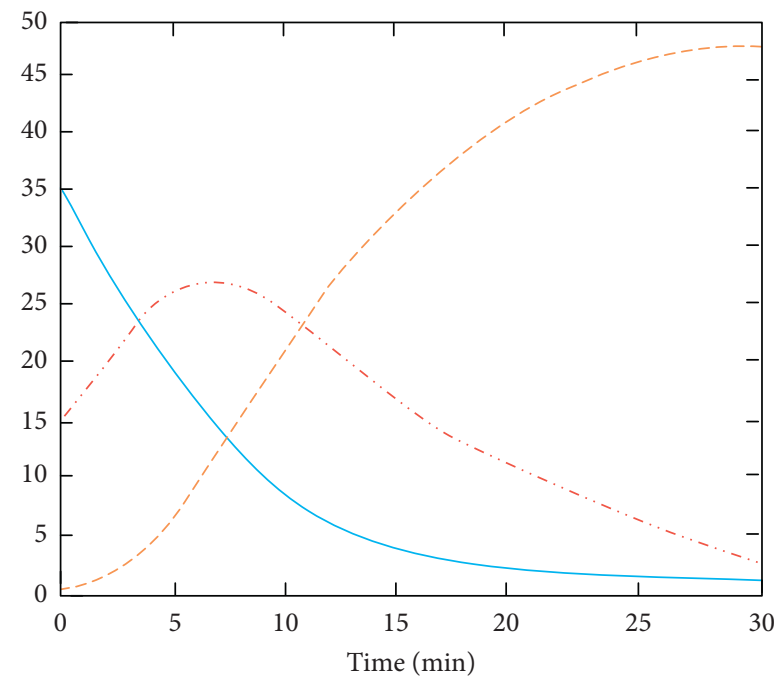

(c)

FiguRE 5: Number of stations for network with multiple delay propagation. (a) 5 stations. (b) 10 stations. (c) 15 stations. “-”: the number of stations that may be delayed (S); “*”: the number of delayed stations (I); “+”: the number of stations where the delay has disappeared (R).

\section{Conclusions}

Analyzing train delays and propagation rule under sudden failure modes can improve the level of refined management of rail transit operations. Single-line faults can propagate to adjacent lines through transfer stations, which can result in delays in the road network to propagate. When the initial delay time is greater than or close to the transfer connection time, the probability of the delay propagating to adjacent lines of the road network greatly increases. The propagation of delays is related to multiple factors such as train departure interval and train running speed. It has a strong linkage effect and plays an important role in the dissipation of delays. The research results of this work can predict the duration and propagation rule of delay in the rail transit network and can evaluate the effect of delay dissipation after failure control measures, in order to provide theoretical and decision support for the formulation of delay dissipation strategies. In the future, we will carry out research on hazard identification and fault fine control around different periods and lines, so as to minimize the impact of train operation delay.

\section{Data Availability}

The data used to support the findings of this study are available from the corresponding author upon request.

\section{Conflicts of Interest}

The author declares that they have no conflicts of interest. 


\section{Acknowledgments}

This work was supported in part by National Key R\&D Program of China under Grant by 2019YFB1600602; Shanghai Committee of Science and Technology under Grant by (18DZ1206300); the National Natural Science Foundation under Grant 52072235; the capacity building project of local colleges of Shanghai Municipal Science and Technology Commission under Grant 19030501400.

\section{References}

[1] Q. Zhang, W. J. Xiao, and G. Pan, "Research on the propagation of rail transit passenger congestion based on cellular automata," Transportation System Engineering and Information, vol. 17, no. 4, pp. 83-89, 2017.

[2] D.-M. Zhang, F. Du, H. Huang, F. Zhang, B. M. Ayyub, and M. Beer, "Resiliency assessment of urban rail transit networks: shanghai metro as an example," Safety Science, vol. 106, pp. 230-243, 2018.

[3] X. Ding, X. Yang, H. Hu, and Z. Liu, "The safety management of urban rail transit based on operation fault log," Safety Science, vol. 94, pp. 10-16, 2017.

[4] Y. Gao, L. Kroon, M. Schmidt, and L. Yang, "Rescheduling a metro line in an over-crowded situation after disruptions," Transportation Research Part B: Methodological, vol. 93, pp. 425-449, 2016.

[5] F. B. Liu, R. H. Xu, and Z. B. Jiang, "Coordinated adjustment method of metro train delay to meet passenger demand," Journal of Tongji University (Natural Science Edition), vol. 2, pp. 215-221, 2018.

[6] R. H. Xu, J. M. Ye, H. C. Pan et al., "Early warning method of large passenger flow at transfer station of urban rail transit network under train operation delay," China Railway Science, vol. 35, no. 5, pp. 127-133, 2014.

[7] X. Chen, X. Xu, Y. Yang et al., "Augmented ship tracking under occlusion conditions from maritime surveillance videos," IEEE Access, vol. 8, pp. 42884-42897, 2020.

[8] K. Qiao and P. Zhao, "Train operation delay of urban rail transit and its adjustment method," Urban Rapid Rail Transit, vol. 26, no. 1, pp. 41-45, 2013.

[9] X. Zhang, Y. Deng, Q. Li, M. Skitmore, and Z. Zhou, “An incident database for improving metro safety: the case of shanghai," Safety Science, vol. 84, pp. 88-96, 2016.

[10] X. Chen, S. Wang, C. Shi, H. Wu, J. Zhao, and J. Fu, "Robust ship tracking via multi-view learning and sparse representation," Journal of Navigation, vol. 72, no. 1, pp. 176-192, 2019.

[11] F. M. Bass, "A new product growth for model consumer durables," Management Science, vol. 15, no. 5, pp. 215-227, 1969.

[12] J. H. Yang, Application Research of Bass Model and its Two Extensions, Dalian University of Technology, Dalian, China, 2006.

[13] M. Carey and A. Kwieciński, "Stochastic approximation to the effects of headways on knock-on delays of trains," Transportation Research Part B: Methodological, vol. 28, no. 4, pp. 251-267, 1994.

[14] T. Büker and B. Seybold, "Stochastic modelling of delay propagation in large networks," Journal of Rail Transport Planning \& Management, vol. 2, no. 1-2, pp. 34-50, 2012.

[15] R. M. P. Goverde, "Railway timetable stability analysis using max-plus system theory," Transportation Research Part B: Methodological, vol. 41, no. 2, pp. 179-201, 2007.
[16] L. E. Meester and S. Muns, "Stochastic delay propagation in railway networks and phase-type distributions," Transportation Research Part B: Methodological, vol. 41, no. 2, pp. 218-230, 2007.

[17] K. Keiji, H. Naohiko, and M. Shigeru, "Simulation analysis of train operation to recover knock-on delay under high-frequency intervals," Case Studies on Transport Policy, vol. 3, no. 1, pp. 92-98, 2015.

[18] L. Yang, K. Li, and Z. Gao, "Train timetable problem on a single-line railway with fuzzy passenger demand," IEEE Transactions on Fuzzy Systems, vol. 17, no. 3, pp. 617-629, 2009.

[19] R. M. P. Goverde, "A delay propagation algorithm for largescale railway traffic networks," Transportation Research Part C: Emerging Technologies, vol. 18, no. 3, pp. 269-287, 2010.

[20] W. Qu, Modeling and Analysis of Urban Rail Transit Train Connection Control Based on Delay Propagation, Beijing Jiaotong University, Beijing, China, 2014.

[21] P. Huang, Q. Y. Peng, C. Wen et al., "Stochastic forest model for predicting train delay recovery time of Wuhan Guangzhou high speed railway," Journal of the China Railway Society, vol. 40, no. 7, pp. 6-14, 2018.

[22] W. Ding, X. H. Liu, and R. Jiang, "Research on Bayesian network model of railway passenger train delay," Journal of Lanzhou Jiaotong University, vol. 32, no. 6, pp. 115-118, 2013.

[23] Y. L. Ye, W. Q. Li, and J. Zhang, "Study on complex characteristics and propagation dynamics of high speed railway network," Journal of Tongji University (Natural Science Edition), vol. 47, no. 5, pp. 67-74, 2019.

[24] L. Wang, Y. Qin, J. Xu et al., "Railway traffic organization under emergency conditions," Journal of Southeast University (Natural Science Edition), vol. 43, no. S1, pp. 12-17, 2013.

[25] Y. Zheng, Research on Metro Operation Accident Delay Model Based on Maximum Likelihood Regression Tree, Beijing Jiaotong University, Beijing, China, 2015.

[26] X. H. Xu, Research on Robustness and Delay Propagation of Aviation Network, Nanjing University of Aeronautics and Astronautics, Nanjing, China, 2018. 